MIFP-08-12

\title{
New Charged Black Holes in Five Dimensions
}

\author{
H. Lü ${ }^{1}$, Jianwei $\mathrm{Mei}^{1}$ and C.N. Pope ${ }^{1}{ }^{2}$ \\ ${ }^{1}$ George P. $\&$ Cynthia Woods Mitchell Institute for Fundamental Physics and Astronomy, \\ Texas A\&M University, College Station, TX 7r843, USA \\ ${ }^{2}$ DAMTP, Centre for Mathematical Sciences, Cambridge University, \\ Wilberforce Road, Cambridge CB3 OWA, UK
}

\begin{abstract}
$\underline{\text { ABSTRACT }}$
We obtain new stationary charged solutions of five-dimensional minimal supergravity. We first obtain purely dipole charged solutions, by extending a technique that we developed for five-dimensional Ricci-flat metrics in a previous paper, which could be viewed as being analogous to a four-dimensional construction by Demianski and Plebanski. The further introduction of electric charge is achieved by means of a solution-generating technique, which exploits the global $S L(2, \mathbb{R})$ symmetry of five-dimensional minimal supergravity reduced on a timelike direction to four dimensions. We present this procedure in detail, since it provides a particularly simple general way of adding charge to any stationary solution of five-dimensional minimal supergravity. The new charged solutions we obtain limit in special cases to black rings carrying electric and magnetic dipole charge, or to charged Myers-Perry rotating black holes. We analyse the general solutions in detail, showing that they can describe asymptotically locally flat black holes whose horizon is a lens space $L(n ; m)=S^{3} / \Gamma(n ; m)$. At infinity they approach Minkowski ${ }_{5} / \Gamma(m ; n)$.
\end{abstract}




\section{Contents}

1 Introduction 1

2 Charging Solutions of Minimal $D=5$ Supergravity 3

3 Solutions with Dipole Charge 6

4 Electrically Charged Dipole Solution 7

5 Global Analysis

6 Conclusions

A 3-Charge Solutions in the STU Model 15

B Black Ring Limit $\quad 16$

B.1 Dipole ring .............................. 17

B.2 Electrically-charged dipole ring . . . . . . . . . . . . . . 19

\section{Introduction}

String theory and M-theory provide a powerful motivation for expanding the long-standing quest for solutions in four-dimensional general relativity to higher dimensions. As well as vacuum solutions of the Einstein equations, solutions including the specific matter fields of the relevant higher-dimensional supergravity theories are also of interest. With the recent discovery of the black ring solutions [1, 2] in five dimensions, some of the previous understanding of black holes and their uniqueness, derived in a four-dimensional setting, needs to be extended.

In a previous paper [3], we looked for higher-dimensional generalisations of the rather large class of four-dimensional Ricci-flat type D metrics that are succinctly described in the form found by Plebanski and Demianski [4. Our starting point was a reformulation [5, 6] of the higher-dimensional rotating black holes, with [7, 8] or without [9] cosmological constant. The reformulation in [5, 6] allowed the natural introduction of additional parameters that could be interpreted as certain higher-dimensional generalisations of the four-dimensional NUT parameter. The structure of the metrics in [5, 6] so closely parallels the four-dimensional Kerr-NUT-de Sitter metrics that one is tempted to expect a parallel "Plebanski-Demianski type" of generalisation in all the higher dimensions too. As was found 
in [10, 3], however, this does not in general seem to be possible. It was shown, however, that five dimensions is rather a special case, and the hoped-for generalisation was indeed found, for Ricci-flat metrics, in that case [3].

The purpose of the present paper is to extend the Ricci-flat results in [3], by constructing analogous solutions of five-dimensional minimal supergravity. This theory, in its bosonic sector (which is all that is relevant for the solutions we shall consider), comprises fivedimensional gravity coupled to a $U(1)$ graviphoton field. The latter is effectively a Maxwell field in five dimensions, together with a Chern-Simons type of $F \wedge F \wedge A$ coupling. The new solutions that we obtain carry both electric charge and also a magnetic dipole charge.

Our strategy for constructing the new solutions is to begin by reformulating the previously known charged rotating black holes of five-dimensional minimal supergravity (contained within the results in [11] or in [12]) in the manner of [5], and then to seek a generalisation analogous to the one found for Ricci-flat metrics in [3]. Owing to the fact that certain Wick rotations are performed in the process, the interpretation of the new metrics at this stage is that they carry not electric charge, but magnetic dipole charge. We then, finally, implement a solution-generating procedure that allows us to introduce a genuine electric charge as well. This solution-generating technique exploits the $S L(2, \mathbb{R})$ global symmetry of five-dimensional minimal supergravity after a timelike reduction to four dimensions. Since it is of rather general utility, and is quite considerably simpler than other presentations of the "charging" procedure that have appeared in the literature, we devote a section of the paper to the description of this procedure in general. It can be applied to any stationary solution of five-dimensional minimal supergravity.

Having obtained the new solutions with electric and magnetic dipole charge we then investigate their global properties. The conditions on the free parameters that must be imposed in order to eliminate conical singularities imply that the spacetime describes a black hole with an horizon that has the topology of a lens space $L(n ; m)$ (a factoring of $S^{3}$ by a certain freely-acting discrete subgroup $\Gamma(n ; m)$ of the isometry group of the sphere). The metrics are asymptotically locally flat, and at large distance they approach five-dimensional Minkowski spacetime factored by $\Gamma(m ; n)$.

Finally, in appendix A, we present a more general class of electrically-charged solutions to $U(1)^{3}$ five-dimensional supergravity. (This theory, sometimes called the STU model, can be thought of as a truncation of maximal supergravity in five dimensions, in which two vector multiplets are coupled to the minimal supergravity.) In appendix B, we consider a certain limit of our general solutions in minimal supergravity, in which the metrics have 
the general form of black rings. The requirement that the solutions in this limit be globally well behaved leads to a bifurcation in the choice of the parameters. In one branch, this corresponds to turning off the electric charge. This solution, which still has a non-vanishing dipole charge, admits choices for the remaining parameters for which it describes a black ring with $S^{2} \times S^{1}$ horizon topology. In the other branch, the electric charge is non-zero but is related to the strength of the dipole field. Again, there exist black ring solutions within this branch. These black ring secialisations are contained within the results found in [13, 14].

\section{Charging Solutions of Minimal $D=5$ Supergravity}

In this section, we present a general procedure for mapping any stationary solution of fivedimensional minimal supergravity into a solution that carries an additional electric charge. Five-dimensional minimal supergravity reduced on 2-torus gives rise to a scalar coset sigma model with $G_{2}$ global symmetry [15, 16]. One way to charge a five-dimensional solution is to reduce it to $D=3$ on the 2 -torus and then "boost" it by acting with an appropriate subgroup of the $G_{2}$ symmetry [17]. However, it was observed in [16] that reducing the theory just on $S^{1}$ gives rise to a $D=4$ theory with an $S L(2, \mathbb{R})$ global symmetry. For our purposes, the charging can be conveniently implemented by performing a timelike reduction to four dimensions, and acting with a certain $O(1,1)$ subgroup of the $S L(2, \mathbb{R})$ duality group. This procedure can be carried out whenever the original five-dimensional solution is stationary.

To describe the procedure, we first reduce five-dimensional minimal supergravity on a timelike Killing vector. In the bosonic sector, the five-dimensional theory is given by

$$
\mathcal{L}_{5}=\hat{R} \hat{*} \mathbb{1}-\frac{1}{2} \hat{*} \hat{F} \wedge \hat{F}+\frac{1}{3 \sqrt{3}} \hat{F} \wedge \hat{F} \wedge \hat{A}
$$

where $\hat{F}=d \hat{A}$, and $\hat{A}$ is the graviphoton potential 1 The stationary metric can be written in the form

$$
d \hat{s}_{5}^{2}=e^{\phi} d s_{4}^{2}-e^{-2 \phi}(d t+\mathcal{A})^{2},
$$

where the quantities on the right-hand side are all independent of $t$.

We now perform a Kaluza-Klein reduction to four dimensions using (2.2), with the reduction of the graviphoton given by

$$
\hat{A}=A+\sqrt{3} \chi(d t+\mathcal{A}) .
$$

\footnotetext{
${ }^{1}$ This implies that the graviphoton equation of motion is $\hat{\nabla}_{\nu} \hat{F}^{\mu \nu}+1 /(4 \sqrt{3}) \epsilon^{\mu \alpha \beta \gamma \delta} \hat{F}_{\alpha \beta} \hat{F}_{\gamma \delta}=0$.
} 
The resulting four-dimensional theory is described by the Lagrangian

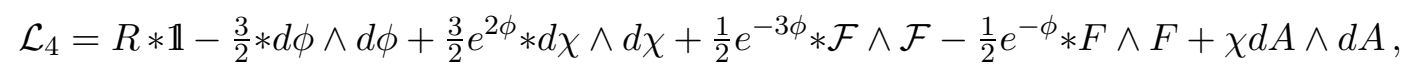

where

$$
\mathcal{F}=d \mathcal{A}, \quad F=d A+\sqrt{3} \chi d \mathcal{A} .
$$

The four-dimensional theory described by (2.4) has an $S L(2, \mathbb{R})$ global symmetry, at the level of the equations of motion. By examining the dilaton coupling of the vector fields, it was observed in [16] that the two vectors and their duals form a quartet representation under the $S L(2, \mathbb{R})$. Here we shall exhibit this structure explicitly. A convenient way to do this is to use a doubled formalism, in which potentials dual to $\mathcal{A}$ and $A$ are introduced.

First, we observe from (2.4) that the equations of motion for $\mathcal{A}$ and $A$ are given by

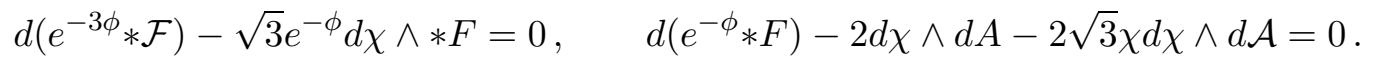

We then define dual fields according to

$$
G=e^{-\phi} * F, \quad \mathcal{G}=e^{-3 \phi} * \mathcal{F} .
$$

It follows from (2.6) that we may write these in terms of dual potentials $B$ and $\mathcal{B}$ as

$$
\begin{aligned}
G & =d B+2 \chi d A+\sqrt{3} \chi^{2} d \mathcal{A} \\
\mathcal{G} & =d \mathcal{B}+\sqrt{3} \chi d B+\sqrt{3} \chi^{2} d A+\chi^{3} d \mathcal{A}
\end{aligned}
$$

We may then derive all the equations of motion and Bianchi identities of the original theory (2.4) from the doubled Lagrangian

$$
\begin{aligned}
\mathcal{L}_{4}= & R * \mathbb{1}-\frac{3}{2} * d \phi \wedge d \phi+\frac{3}{2} e^{2 \phi} * d \chi \wedge d \chi+\frac{1}{4} e^{-3 \phi_{*}} \mathcal{F} \wedge \mathcal{F}-\frac{1}{4} e^{3 \phi} * \mathcal{G} \wedge \mathcal{G} \\
& -\frac{1}{4} e^{-\phi_{*}} F \wedge F+\frac{1}{4} e^{\phi} * G \wedge G,
\end{aligned}
$$

together with (2.7).

To make the $S L(2, \mathbb{R})$ symmetry manifest, we define the $S L(2, \mathbb{R}) / O(1,1)$ scalar coset representative $\mathcal{V}$ and the matrix $\mathcal{M}$

$$
\mathcal{V}=\left(\begin{array}{cc}
e^{\frac{1}{2} \phi} & e^{\frac{1}{2} \phi} \chi \\
0 & e^{-\frac{1}{2} \phi}
\end{array}\right), \quad \mathcal{M}=\mathcal{V}^{T} \eta \mathcal{V}, \quad \eta=\left(\begin{array}{cc}
-1 & 0 \\
0 & 1
\end{array}\right)
$$

and we also define the $S L(2, \mathbb{R})$ quartet of 1 -form potentials $\mathbf{A}^{\alpha \beta \gamma}$, which is symmetric in its three $S L(2, \mathbb{R})$ doublet indices $\alpha, \beta$ and $\gamma$, by

$$
\mathbf{A}^{111}=\mathcal{A}, \quad \mathbf{A}^{112}=-\frac{1}{\sqrt{3}} A, \quad \mathbf{A}^{122}=\frac{1}{\sqrt{3}} B, \quad \mathbf{A}^{222}=-\mathcal{B} .
$$


It can then be seen that the Lagrangian (2.9) may be written as

$$
\mathcal{L}=R * \mathbb{1}+\frac{3}{4} \operatorname{tr}\left(* d \mathcal{M}^{-1} \wedge d \mathcal{M}\right)-\frac{1}{4} * d \mathbf{A}^{\alpha \beta \gamma} \wedge d \mathbf{A}^{\delta \sigma \tau}\left(\mathcal{M}^{-1}\right)_{\alpha \delta}\left(\mathcal{M}^{-1}\right)_{\beta \sigma}\left(\mathcal{M}^{-1}\right)_{\gamma \tau}
$$

Defining

$$
\mathbf{F}^{\alpha \beta \gamma} \equiv\left(\mathcal{V}^{-1}\right)_{\delta}^{\alpha}\left(\mathcal{V}^{-1}\right)_{\sigma}^{\beta}\left(\mathcal{V}^{-1}\right)_{\tau}^{\gamma} d \mathbf{A}^{\delta \sigma \tau}
$$

the duality relations (2.7) can be written as $\mathbf{F}^{222}=-* \mathbf{F}^{111}$ and $\mathbf{F}^{122}=-* \mathbf{F}^{112}$. They can also be recast as

$$
d \mathbf{A}^{\alpha_{1} \alpha_{2} \alpha_{3}}=-\mathcal{M}^{\alpha_{1} \beta_{1}} \mathcal{M}^{\alpha_{2} \beta_{2}} \mathcal{M}^{\alpha_{3} \beta_{3}} \epsilon_{\beta_{1} \gamma_{1}} \epsilon_{\beta_{2} \gamma_{2}} \epsilon_{\beta_{3} \gamma_{3}} * d \mathbf{A}^{\gamma_{1} \gamma_{2} \gamma_{3}}
$$

The Lagrangian (2.12) and the duality relation (2.14) are manifestly invariant under the $S L(2, \mathbb{R})$ transformations

$$
\mathcal{M} \longrightarrow \Lambda^{T} \mathcal{M} \Lambda, \quad \mathbf{A}^{\alpha \beta \gamma} \longrightarrow \Lambda_{\delta}^{\alpha} \Lambda_{\sigma}{ }^{\beta} \Lambda_{\tau}^{\gamma} \mathbf{A}^{\delta \sigma \tau}
$$

where

$$
\Lambda=\left(\begin{array}{ll}
a & b \\
c & d
\end{array}\right), \quad a d-b c=1 .
$$

We are interested in the action of the $O(1,1)$ subgroup of $S L(2, \mathbb{R})$ transformations

$$
\Lambda=\left(\begin{array}{ll}
c & s \\
s & c
\end{array}\right)
$$

where $c \equiv \cosh \delta$ and $s \equiv \sinh \delta$, on the fields $\phi, \chi, \mathcal{A}$ and $A$ of the dimensionally-reduced original solution. The $O(1,1)$-transformed (primed) fields are therefore given by

$$
\begin{aligned}
\epsilon^{\phi} & \longrightarrow e^{\phi^{\prime}}=e^{\phi}(c+s \chi)^{2}-s^{2} e^{-\phi}, \\
\chi & \longrightarrow \chi^{\prime}=\frac{e^{\phi}(c+s \chi)(s+c \chi)-s c e^{-\phi}}{e^{\phi}(c+s \chi)^{2}-s^{2} e^{-\phi}}, \\
\mathcal{A} & \longrightarrow \mathcal{A}^{\prime}=c^{3} \mathcal{A}-\sqrt{3} c^{2} s A+\sqrt{3} c s^{2} B-s^{3} \mathcal{B}, \\
A & \longrightarrow A^{\prime}=-\sqrt{3} c^{2} s \mathcal{A}+c\left(3 c^{2}-2\right) A+\left(1-3 c^{2}\right) s B+\sqrt{3} c s^{2} \mathcal{B} .
\end{aligned}
$$

Having obtained the transformed four-dimensional fields, we then substitute these quantities into (2.2) and (2.3) in order to obtain the new solution of the minimal five-dimensional supergravity equations. (We have not listed the transformed dual potentials $B$ and $\mathcal{B}$ in (2.18), since they are not needed in the lifting back to $D=5$.)

In section 3, we construct a new dipole-charged solution of minimal five-dimensional supergravity, and then in section 4, we apply the procedure we have just described in order to construct an electrically charged generalisation. 


\section{Solutions with Dipole Charge}

In this section, we construct a new solution of the equations of motion of five-dimensional minimal supergravity, which generalises the charged Myers-Perry solution that is contained in the results of [11, 12]. The new solution that we construct here is analogous to the generalisation of the neutral five-dimensional Myers-Perry black hole that we obtained in [3].

Although the solution we shall construct in this section arises as a generalisation of the electrically-charged version of the Myers-Perry solution, it will be natural, from the point of view we are adopting here, to reinterpret the ignorable coordinates in such a way that it carries not an electric charge, but instead a magnetic dipole charge. This new dipole-charged solution will then itself be subjected, in section 4 , to the "charging" procedure described in section 2 .

We take as our starting point the electrically-charged rotating black hole solution of five-dimensional minimal supergravity, which we find can be written as

$$
\begin{aligned}
d s^{2}= & (x-y)\left(\frac{d x^{2}}{4 X}-\frac{d y^{2}}{4 Y}\right)-\frac{X(d t+y d \phi)^{2}}{x(x-y)}+\frac{Y(d t+x d \phi)^{2}}{y(x-y)} \\
& -\frac{1}{x y}\left[\left(\mu-\frac{q y}{x-y}\right) d t+(x+y)\left(\mu-\frac{q y^{2}}{x^{2}-y^{2}}\right) d \phi+x y d \chi\right]^{2} \\
A= & \frac{\sqrt{3} q}{(x-y)}(d t+y d \phi)
\end{aligned}
$$

where

$$
X=(\mu+q)^{2}+a_{3} x+a_{2} x^{2}, \quad Y=\mu^{2}+a_{1} y+a_{2} y^{2} .
$$

This is actually a special case of the charged rotating black holes found in [11, in which the three $U(1)$ charges are set equal. The form in which the solution is written in (3.1) is, however, considerably simpler. If the charge parameter $q$ is set to zero, this solution reduces to the five-dimensional Myers-Perry [9] rotating black hole, in the simpler form introduced in [5].

Our strategy now is analogous to the one we followed in [3], in which we obtained a generalisation of the $q=0$ five-dimensional rotating black hole by introducing a suitable conformal factor multiplying a four-dimensional subspace (the projection of the metric in (3.1) that is orthogonal to $\partial / \partial t$ ), and at the same time adjusting the detailed forms of the metric functions $X(x)$ and $Y(y)$. In order to do this, it will be convenient first to make the coordinate redefinitions

$$
t \longrightarrow \phi, \quad \phi \longrightarrow \psi, \quad \chi \longrightarrow t, \quad x \longrightarrow \frac{1}{x}
$$


in the solution (3.1).

Introducing a conformal factor, and imposing the equations of motion following from (2.1), we obtain the new solution

$$
\begin{aligned}
d s^{2}= & \frac{1}{(x-y)^{2}}\left[x(1-x y)\left(\frac{d x^{2}}{4 G(x)}-\frac{d y^{2}}{4 G(y)}\right)-\frac{G(x)(d \phi+y d \psi)^{2}}{1-x y}+\frac{x G(y)(d \psi+x d \phi)^{2}}{y(1-x y)}\right] \\
& -\frac{y}{x}\left[d t+\frac{x}{y}\left(\mu-\frac{q x y}{1-x y}\right) d \phi+\left(x+y^{-1}\right)\left(\mu-\frac{q x^{2} y^{2}}{1-x^{2} y^{2}}\right) d \psi\right]^{2} \\
A= & \frac{\sqrt{3} q x}{1-x y}(d \phi+y d \psi),
\end{aligned}
$$

where

$$
G(\xi)=\mu^{2}+a_{1} \xi+a_{2} \xi^{2}+a_{3} \xi^{3}+(\mu+q)^{2} \xi^{4} .
$$

In appendix B, we shall discuss a limit of this new solution in which it reduces to a black ring. We also note here, in passing, that we can take a different limit in which the solution reduces again to the charged Myers-Perry black hole. In this limit, the $x$ and $y$ coordinates in (3.4) are subjected to inverse scaling transformations, with

$$
\begin{aligned}
& x \longrightarrow \frac{1}{\epsilon^{2} x}, \quad y \longrightarrow \epsilon^{2} y, \quad \phi \longrightarrow \epsilon^{-1} t, \quad t \longrightarrow \epsilon^{-2} \chi, \quad \psi \longrightarrow \phi, \\
& \mu \longrightarrow \epsilon^{3} \mu, \quad q \longrightarrow \epsilon^{3} q, \quad a_{1} \longrightarrow \epsilon^{4} a_{1}, \quad a_{2} \longrightarrow \epsilon^{2} a_{2}, \quad a_{3} \longrightarrow \epsilon^{4} a_{3},
\end{aligned}
$$

and $\epsilon$ then sent to zero. The solution reduces to (3.1).

\section{Electrically Charged Dipole Solution}

Having obtained the local solution (3.4) that is supported by purely magnetic dipole-like charges, we can apply the procedure described in section 2 in order to introduce electric charge. This is achieved by first reducing the solution on the time direction, performing an $O(1,1)$ U-duality transformation, and then lifting the solution back to $D=5$. By this means we obtain the new solution

$$
\begin{aligned}
d s_{5}^{2}= & H d s_{4}^{2}-\frac{y}{x H^{2}}(d t+\omega)^{2}, \\
\omega= & -\frac{q(s+c x)^{3}(d \phi+y d \psi)}{x(1-x y)}+\frac{(\mu+q) s^{3}((1+x y) d \phi+y d \psi)}{x} \\
& +\frac{c^{3} \mu((1+x y) d \psi+x d \phi)}{y}, \\
A= & \frac{\sqrt{3}}{x H}\left(c s(x-y) d t+\frac{q(s+c x)^{2}(c+s y)(d \phi+y d \psi)}{(1-x y)}\right. \\
& \left.-(\mu+q) c s^{2}((1+x y) d \phi+y d \psi)-\mu c^{2} s((1+x y) d \psi+x d \phi)\right), \\
H= & c^{2}-\frac{s^{2} y}{x}
\end{aligned}
$$


where $d s_{4}^{2}$ is the same as the four-dimensional base metric in (3.4), namely

$$
d s_{4}^{2}=\frac{1}{(x-y)^{2}}\left[x(1-x y)\left(\frac{d x^{2}}{4 G(x)}-\frac{d y^{2}}{4 G(y)}\right)-\frac{G(x)(d \phi+y d \psi)^{2}}{1-x y}+\frac{x G(y)(d \psi+x d \phi)^{2}}{y(1-x y)}\right],
$$

and the function $G(\xi)$ is again given by (3.5):

$$
G(\xi)=\mu^{2}+a_{1} \xi+a_{2} \xi^{2}+a_{3} \xi^{3}+(\mu+q)^{2} \xi^{4}
$$

\section{Global Analysis}

In this section, we shall discuss the global properties of the new charged solutions (4.1) that we have obtained. It is advantageous to reparameterise the constants in the function $G(\xi)$ so that it is written as

$$
G(\xi)=(\mu+q)^{2}\left(\xi-\xi_{1}\right)\left(\xi-\xi_{2}\right)\left(\xi-\xi_{3}\right)\left(\xi-\xi_{4}\right)
$$

with $\xi_{1} \xi_{2} \xi_{3} \xi_{4}=\mu^{2} /(\mu+q)^{2}$. Furthermore, we let $\xi_{1}<\xi_{2}<0<\xi_{3}<\xi_{4}$, with $\xi_{1} \xi_{2}<1$. As we shall see below, there exist black hole solutions in which the region outside the horizon is covered by the coordinate ranges

$$
\xi_{1} \leq x \leq \xi_{2}, \quad \xi_{2} \leq y \leq \xi_{3}
$$

Asymptotic infinity is located at $x=\xi_{2}=y$, and the horizon is at $y=\xi_{3}$, with an ergosphere at $y=0$. For later convenience we shall parameterise the two negative roots $\xi_{1}$ and $\xi_{2}$ in terms of the positive constants

$$
\eta_{1} \equiv-\xi_{1}, \quad \eta_{2} \equiv-\xi_{2}
$$

The global analysis that we shall give in this section closely parallels the one that we described in [3] where new uncharged five-dimensional black holes were obtained.

It is useful at this stage to shift the time coordinate $t$ in (4.1) according to

$$
t \rightarrow t-\left(3 c^{2} s q-c^{3}(\mu+q)\left(\eta_{1}+\eta_{2}\right)+\frac{\mu s^{3}}{\eta_{1} \eta_{2}}\right) \psi+\left(3 c s^{2} q-c^{3}(\mu+q) \eta_{1} \eta_{2}+\frac{\mu s^{3}\left(\eta_{1}+\eta_{2}\right)}{\eta_{1} \eta_{2}}\right) \phi
$$

In order to avoid naked CTCs, we find that the parameters in the solution should be chosen so that

$$
q=\frac{\mu\left(s^{3}+c^{3} \eta_{1}\right)\left(1-\eta_{1} \eta_{2}\right)\left(1-\eta_{2}^{2}\right)}{\eta_{1} \eta_{2}\left(c^{3}\left(\eta_{1}+\eta_{2}-\eta_{1} \eta_{2}^{2}\right)-s\left(3 c^{2}-3 c s \eta_{2}+s^{2} \eta_{2}^{2}\right)\right)} .
$$

Note that if we turn off $q$, we can have either $\eta_{1} \eta_{2}=1$ or $\eta_{1}=-s^{3} / c^{3}$. The former case leads to a charged rotating black hole solution, whilst in the latter case, a conical singularity 
cannot be avoided for finite $s$ or $c$, since $\eta_{1}$ would be less than zero, as will become apparent presently.

The analysis of the global properties of the solution rests upon investigating the behaviour at the singular points of the metric, which occur when $x$ or $y$ approach their endpoints (see (5.2)). We shall follow the method of analysis that was introduced in [18], which begins by writing down the (appropriately normalised) Killing vectors whose norms tend to zero at the spacelike degeneration surfaces. In the present case, these degenerations occur at $x=\xi_{1}, x=\xi_{2}$ and $y=\xi_{2}$. The associated Killing vectors will be normalised so that they have unit Euclidean surface gravity. (The Euclidean surface gravity $\kappa_{E}$ of a Killing vector $K$ whose norm goes to zero on a spacelike degeneration surface is given by $\kappa_{E}^{2}=\lim \left(g^{\mu \nu}\left(\partial_{\mu} K^{2}\right)\left(\partial_{\nu} K^{2}\right) /\left(4 K^{2}\right)\right)$.)

A redefinition of azimuthal coordinates is helpful at this stage. We introduce $\phi_{1}$ and $\phi_{2}$, in place of $\phi$ and $\psi$, defined by

$$
\begin{aligned}
& \psi=\nu\left(\eta_{2} \phi_{1}-\phi_{2}\right), \quad \phi=\nu\left(\phi_{1}-\eta_{2} \phi_{2}\right), \\
& \nu \equiv \frac{\sqrt{\eta_{2}}}{(\mu+q)^{2}\left(\eta_{2}+\xi_{3}\right)\left(\eta_{2}+\xi_{4}\right)\left(\eta_{1}-\eta_{2}\right)} .
\end{aligned}
$$

(Recall that $\eta_{1}$ and $\eta_{2}$ are defined by (5.3) .) We then find that the normalised Killing vectors at the three degeneration surfaces are given by

$$
\begin{array}{ll}
x=\xi_{1}=-\eta_{1}: & \ell_{1}=\alpha\left(\left(1-\eta_{1} \eta_{2}\right) \frac{\partial}{\partial \phi_{1}}-\left(\eta_{1}-\eta_{2}\right) \frac{\partial}{\partial \phi_{2}}\right), \\
y=\xi_{2}=-\eta_{2}: & \ell_{2}=\frac{\partial}{\partial \phi_{2}} \\
x=\xi_{2}=-\eta_{2}: & \ell_{3}=\frac{\partial}{\partial \phi_{1}},
\end{array}
$$

where the constant $\alpha$ is given by

$$
\alpha=\frac{\sqrt{\eta_{1}}\left(\eta_{2}+\xi_{3}\right)\left(\eta_{2}+\xi_{4}\right)}{\sqrt{\eta_{2}}\left(\eta_{1}+\xi_{3}\right)\left(\eta_{1}+\xi_{4}\right)\left(1-\eta_{2}^{2}\right)} .
$$

Because of the chosen normalisation, if each Killing vector $\ell_{i}$ is written in terms of a coordinate $\psi_{i}$ as $\ell_{i}=\partial / \partial \psi_{i}$, then advancing $\psi_{i}$ by an interval $2 \pi$ will generate one complete rotation around the origin in the plane of the degeneration (just like the standard azimuthal angle in polar coordinates on the Euclidean 2-plane).

The three Killing vectors (5.7) span a two-dimensional vector space, and so there is a linear relation between them. A necessary condition for avoiding conical singularities is that the coefficients in this linear relation must be rationally related, since otherwise it would be possible, by taking integer combinations of $2 \pi$ rotations around the circles, to generate a 
translation that implied an identification of arbitrarily close points on the manifold. By an overall scaling in the linear relation, we may therefore say, without loss of generality, that the coefficients must be coprime integers $m, n$ and $p$,

$$
p \ell_{3}=m \ell_{1}+n \ell_{2}
$$

The Killing vectors $\ell_{1}$ and $\ell_{2}$ can degenerate simultaneously, and, as discussed in [18], this implies an additional condition, leading to the constraint

$$
\ell_{3}=m \ell_{1}+n \ell_{2}
$$

It therefore follows from (5.7) that the coprime integers $(m, n)$ are given by

$$
m=\frac{1}{\alpha\left(1-\eta_{1} \eta_{2}\right)}, \quad n=\frac{\eta_{1}-\eta_{2}}{1-\eta_{1} \eta_{2}} .
$$

Note that we therefore have

$$
\ell_{1}=\frac{1}{m} \frac{\partial}{\partial \phi_{1}}-\frac{n}{m} \frac{\partial}{\partial \phi_{2}} .
$$

It is helpful to look at the double degeneration, at $x=\xi_{1}$ and $y=\xi_{2}$, in more detail. Introducing new coordinates $\rho$ and $\vartheta$, we write

$$
x=-\eta_{1}+e_{1} \rho^{2} \sin ^{2} \vartheta, \quad y=-\eta_{2}+e_{2} \rho^{2} \cos ^{2} \vartheta
$$

where

$$
e_{1}=\left(\eta_{1}+\xi_{3}\right)\left[\mu^{2}+(\mu+q)^{2} \eta_{1}^{2} \eta_{2} \xi_{3}\right], \quad e_{2}=\left(\eta_{2}+\xi_{3}\right)\left[\mu^{2}+(\mu+q)^{2} \eta_{1} \eta_{2}^{2} \xi_{3}\right]
$$

and then look at the metric (4.1) in the limit when $\rho$ is small. It is convenient first to make a further change of azimuthal coordinates, introducing $\chi_{1}$ and $\chi_{2}$ given by

$$
\chi_{1}=m \phi_{1}, \quad \chi_{2}=\phi_{2}+n \phi_{1}
$$

We then find that near $\rho=0$, the metric (4.1) approaches

$$
\begin{aligned}
d s^{2}= & \frac{\eta_{1} \eta_{2}\left(1-\eta_{1} \eta_{2}\right)\left(c^{2} \eta_{1}-s^{2} \eta_{2}\right) \xi_{3}}{\left(\eta_{1}-\eta_{2}\right)^{3}}\left(d \rho^{2}+\rho^{2}\left[d \vartheta^{2}+\sin ^{2} \vartheta d \chi_{1}^{2}+\cos ^{2} \vartheta d \chi_{2}^{2}\right]\right) \\
& -\frac{\eta_{1} \eta_{2}}{\left(c^{2} \eta_{1}-s^{2} \eta_{2}\right)^{2}} d t^{2}
\end{aligned}
$$

This shows that to avoid the occurrence of conical singularities, $\chi_{1}$ and $\chi_{2}$ must have independent $2 \pi$ periods (so that the collapsing $\rho=$ constant surfaces are 3 -spheres with no identifications). Thus $\chi_{1}$ and $\chi_{2}$ are periodic on a square lattice of side $2 \pi$.

One might think that there would be another double degeneration surface at $x=y=\xi_{2}$, leading to another restriction on the periods, but in fact this region actually describes 
asymptotic infinity. To make thid manifest, we introduce new coordinates $r$ and $\theta$, in terms of which we write

$$
x=-\eta_{2}-\frac{\sqrt{\eta_{2}}\left(1-\eta_{2}^{2}\right) \nu \cos ^{2} \theta}{r^{2}}, \quad y=-\eta_{2}+\frac{\sqrt{\eta_{2}}\left(1-\eta_{2}^{2}\right) \nu \sin ^{2} \theta}{r^{2}}
$$

In the limit $r \rightarrow \infty$, we then find that the metric (4.1) approaches

$$
d s^{2}=-d t^{2}+d r^{2}+r^{2}\left(d \theta^{2}+\cos ^{2} \theta d \phi_{1}^{2}+\sin ^{2} \theta d \phi_{2}^{2}\right)
$$

Since from (15.15) we have

$$
\phi_{1}=\frac{1}{m} \chi_{1}, \quad \phi_{2}=\chi_{2}-\frac{n}{m} \chi_{1}
$$

and since we have already determined that $\chi_{1}$ and $\chi_{2}$ are periodic on a square lattice of side $2 \pi$, it follows that $\phi_{1}$ and $\phi_{2}$ are periodic on as tilted lattice, which may be defined by the two identifications

$$
\begin{array}{ll}
\text { 1) } & \phi_{1} \longrightarrow \phi_{1}, \quad \phi_{2} \longrightarrow \phi_{2}+2 \pi, \\
\text { 2) } & \phi_{1} \longrightarrow \phi_{1}+\frac{2 \pi}{m}, \quad \phi_{2} \longrightarrow \phi_{2}-\frac{2 \pi n}{m} .
\end{array}
$$

These identifications imply that the $r=$ constant surfaces in (5.18) are the lens space $L(m ; n)$. This is defined by considering complex coordinates $\left(z_{1}, z_{2}\right)$, in terms of which the unit $S^{3}$ is described by $\left|z_{1}\right|^{2}+\left|z_{2}\right|^{2}=1$. The sphere is then quotiented by the identifications

$$
\left(z_{1}, z_{2}\right) \longrightarrow\left(z_{1} e^{2 \pi \mathrm{i} / m}, z_{2} e^{2 \pi \mathrm{in} / m}\right)
$$

for coprime integers $m$ and $n$ with $1 \leq n \leq m-1$, to define the lens space $L(m ; n)$. We may take (5.21) to define the discrete subgroup $\Gamma(m ; n)$ of $S O(4)$. By taking $z_{1}=\sin \theta e^{\mathrm{i} \phi_{1}}$ and $z_{2}=\cos \theta e^{-\mathrm{i} \phi_{2}}$, it can be seen that (5.21) implies (5.20). In turn, this implies that the $r=$ constant surfaces at large $r$ are lens space $L(m ; n)=S^{3} / \Gamma(m ; n)$.

The event horizon is located at $y=\xi_{3}$. In order to study the geometry and topology of the horizon, it is helpful to introduce a yet further new pair of azimuthal coordinates $\tilde{\phi}_{1}$ and $\tilde{\phi}_{2}$, defined by

$$
\tilde{\phi}_{1}=\frac{1}{n} \chi_{2}=\phi_{1}+\frac{1}{n} \phi_{2}, \quad \tilde{\phi}_{2}=\chi_{1}-\frac{m}{n} \chi_{2}=-\frac{m}{n} \phi_{2} .
$$

In terms of these, the Killing vectors $\ell_{1}$ and $\ell_{3}$ in (5.7), which degenerate at $x=\xi_{1}$ and $x=\xi_{2}$ respectively, are simply given by

$$
\ell_{1}=\frac{\partial}{\partial \tilde{\phi}_{2}}, \quad \ell_{2}=\frac{\partial}{\partial \tilde{\phi}_{1}} .
$$


Writing $x=\xi_{1}+\rho_{1}^{2}$ or $x=\xi_{2}-\rho_{2}^{2}$, for small $\rho_{i}$, to describe the regions near the two degeneration surfaces, we find that the metric on the horizon has the form

$$
d s_{H}^{2} \sim b_{1}\left(d \rho_{1}^{2}+\rho_{1}^{2} d \tilde{\phi}_{2}^{2}\right)+c_{1}\left(d \tilde{\phi}_{1}+\mathcal{A}_{1}\right)^{2}
$$

near $x=\xi_{1}$ and

$$
d s_{H}^{2} \sim b_{2}\left(d \rho_{2}^{2}+\rho_{2}^{2} d \tilde{\phi}_{1}^{2}\right)+c_{2}\left(d \tilde{\phi}_{2}+\mathcal{A}_{2}\right)^{2}
$$

near $x=\xi_{2}$, where the $b_{i}$ and $c_{i}$ are non-singular and non-zero. The 1 -forms $\mathcal{A}_{i}$ are of the form $\mathcal{A}_{1}=\rho_{1}^{2} f_{1} d \tilde{\phi}_{2}+g_{1} d t$ and $\mathcal{A}_{2}=\rho_{2}^{2} f_{2} d \tilde{\phi}_{1}+g_{2} d t$, where $f_{i}$ and $g_{i}$ are non-singular. It is straightforward to see that, in a manner quite analogous to the situation for static lens-space black holes in [3], the horizon therefore has the local geometry of a distorted 3-sphere. As in [3], the relation between $\left(\tilde{\phi}_{1}, \tilde{\phi}_{2}\right)$ and $\left(\chi_{1}, \chi_{2}\right)$ is just like the relation between $\left(\phi_{1}, \phi_{2}\right)$ and $\left(\chi_{1}, \chi_{2}\right)$, except that the roles of the integers $m$ and $n$ are reversed. This means that the identifications of the $\tilde{\phi}_{i}$ coordinates imply that the topology of the horizon is $S^{3} / \Gamma(n ; m)$, or, in other words, the lens space $L(n ; m)$.

By studying the behaviour near infinity, we can determine the mass and angular momenta by means of the Komar integrals $M=3 /(32 \pi) \int * d K[t]$ and $J_{\phi_{i}}=1 /(8 \pi) \int * d K\left[\phi_{i}\right]$, where $K[t]$ and $K\left[\phi_{i}\right]$ are the 1 -forms associated with $\partial / \partial t$ and $\partial / \partial \phi_{i}$. They, and the electric charge $Q_{e}=1 /(16 \pi) \int(* F-1 / \sqrt{3} A \wedge F)$ are given by

$$
\begin{aligned}
M= & \frac{3 \pi\left(1+2 s^{2}\right)\left(1-\eta_{2}^{2}\right) \nu}{8 m \sqrt{\eta_{2}}}, \quad Q_{e}=\frac{\sqrt{3} \pi s c\left(1-\eta_{2}^{2}\right) \nu}{4 m \sqrt{\eta_{2}}} \\
J_{\phi_{1}}= & \frac{\pi \nu^{2}}{4 m \eta_{2}^{3 / 2}}\left(\mu\left(1-\eta_{2}^{2}\right)^{2}\left(s^{3}+c^{3} \eta_{2}\right)+q \eta_{2}^{2}\left(3 c^{2} s-c\left(2+5 s^{2}\right) \eta_{2}+s^{3} \eta_{2}^{2}+c^{3} \eta_{2}^{3}\right)\right) \\
J_{\phi_{2}}= & \frac{\mu \pi\left(1-\eta_{2}^{2}\right) \nu^{2}}{4 m \eta_{1} \eta_{2}^{3 / 2}\left(3 c^{2} s-3 c s^{2} \eta_{2}+s^{3} \eta_{2}^{2}-c^{3}\left(\eta_{1}+\eta_{2}-\eta_{1} \eta_{2}^{2}\right)\right)}\left(c^{3} s^{3}\left(\eta_{1}-\eta_{2}\right)^{2} \eta_{2}\right. \\
& +s^{2} \eta_{2}\left(3 c^{4} \eta_{1}-s^{4} \eta_{2}\right)\left(2-\eta_{1} \eta_{2}-\eta_{2}^{2}\right)+c^{2}\left(c^{4} \eta_{1}-3 s^{4} \eta_{2}\right)\left(\eta_{1}+\eta_{2}-2 \eta_{1} \eta_{2}^{2}\right) \\
& \left.-3 c s\left(c^{4} \eta_{1}-s^{4} \eta_{2}\right)\left(1-\eta_{1} \eta_{2}^{3}\right)\right) .
\end{aligned}
$$

The horizon is located at $y=\xi_{3}$, which is topologically a lens space $L(n ; m)$. The temperature, entropy and angular velocities and electric potentials can be easily calculated, and 
are given by

$$
\begin{aligned}
S & =\frac{\pi^{2}\left(\eta_{1}-\eta_{2}\right)\left(1-\eta_{2}^{2}\right) \nu^{2} \beta}{2 m \sqrt{\xi_{3}} \eta_{1} \eta_{2}\left(\eta_{1}+\xi_{3}\right)\left(\eta_{2}+\xi_{3}\right)} \\
T & =\frac{1}{2 \pi \beta}(\mu+q)^{2} \sqrt{\xi_{3}} \eta_{1} \eta_{2}\left(\eta_{1}+\xi_{3}\right)\left(\eta_{2}+\xi_{3}\right)\left(\xi_{4}-\xi_{3}\right) \\
\Omega_{\phi_{1}}= & \frac{\eta_{1} \eta_{2} \xi_{3}\left(\xi_{3}+\eta_{2}\right)}{\nu \beta\left(1-\eta_{2}^{2}\right)}, \quad \Omega_{\phi_{2}}=\frac{\eta_{1} \eta_{2} \xi_{3}\left(1+\eta_{2} \xi_{3}\right)}{\nu \beta\left(1-\eta_{2}^{2}\right)} \\
\Phi_{e}= & \frac{\sqrt{3} \eta_{1}\left(\eta_{2}+\xi_{3}\right)}{\beta\left(1-\eta_{2}^{2}\right)}\left(\mu c s\left(c-s \xi_{3}\right)\left(1-\eta_{2}^{2}\right)\left(1+\eta_{2} \xi_{3}\right)\right. \\
& \left.\quad-q \eta_{2} \xi_{3}\left[-c \eta_{2}+s\left(2+\eta_{2}^{2}\right)+s^{3}\left(3+\eta_{2}^{2}\right)+c s^{2}\left(\xi_{3}-4 \eta_{2}-\eta_{2}^{2} \xi_{3}\right)\right]\right)
\end{aligned}
$$

where

$$
\begin{aligned}
\beta= & \mu\left(1+\eta_{1} \xi_{3}\right)\left(1+\eta_{2} \xi_{3}\right)\left(c^{3} \eta_{1} \eta_{2}-s^{3} \xi_{3}\right) \\
& -q \eta_{1} \eta_{2} \xi_{3}\left(3 c^{2} s+3 c s^{2} \xi_{3}+s^{3} \xi_{3}^{2}-c^{3}\left(\eta_{1}+\eta_{2}+\eta_{1} \eta_{2} \xi_{3}\right)\right),
\end{aligned}
$$

\section{Conclusions}

In this paper, we have constructed new charged solutions of five-dimensional supergarvity, which generalise the new Ricci-flat metrics that we obtained in [3]. To do this, we first established a procedure, utilising the global $S L(2, \mathbb{R})$ symmetry of the four-dimensional theory obtained from a timelike reduction of five-dimensional minimal supergravity, which enabled us to introduce electric charge in any stationary solution of the five-dimensional theory. This simple prodedure, described in section 2. is of quite general utility, and has applications beyond those in this paper.

We then constructed the "seed" solution in five-dimensional minimal supergravity, which forms the starting point for the charging proceedure. The starting point for the construction of the seed solution was the electrically-charged generalisation of the five-dimensional rotatating Myers-Perry black hole; this is a specialisation (to minimal supergravity) of more general charged solutions obtained in [11]. After a reinterpretation of the coordinates, this solution can be viewed as carrying a magnetic dipole charge, rather than an electric charge. Next, we looked for a generalisation of this solution, along the same lines as the generalisation of Ricci-flat metrics that allowed us to construct new vacuum solutions in [3]. This involved the introduction of a conformal factor for a four-dimensional base. This provided us with new rotating solutions of minimal five-dimensional supergravity, carrying magnetic dipole charge. These formed the seed solutions to which we then applied the charging procedure. 
After charging the seed solution, we arrived at our new solution (4.1), which carries both electric charge and magnetic dipole charge. A special limit of the solution gives back the charged rotating black hole in five-dimensional minimal supergravity. In another limit, the solution reduces to a class of black rings found in [13, 14. We discussed this limit in detail in appendix B,

In the remainder of the body of the paper, we analysed the global proprties of the general solution (4.1). We found that provided the parameters are chosen properly, with two algebraic conditions characterised by coprime integers $m$ and $n$, the solution describes a stationary black hole spacetime that is asymptotically locally flat, with an horizon that is topologically the lens space $L(n ; m)=S^{3} / \Gamma(n ; m)$. At large distance, the spacetime approaches (Minkowski) $)_{5} / \Gamma(m ; n)$. The two algebraic conditions involving $m$ and $n$ arose from requiring that the spacetime be free from conical singularities.

Our results in this paper and in [3] have all been restricted to five dimensions, and to the case where there is no cosmological constant. Generalisations beyond five dimensions, and also generalisations with a cosmological constant or a gauge coupling in supergravity, would be of considerable interest. Such solutions might admit limits that could describe higher-dimensional black holes with ring-like topologies, or black rings in asymptotically AdS backgrounds.

\section{Acknowledgements}

We are grateful to Gary Gibbons and Harvey Reall for helpful discussions. H.L. is grateful to ICTS at the University of Science and Technology of China, and C.N.P. is grateful to the Relativity and Cosmology groups at the Centre for Mathematical Sciences, Cambridge, for hospitality during the course of this work. Research is supported in part by DOE grant DE-FG03-95ER40917. 


\section{Appendices}

\section{A 3-Charge Solutions in the STU Model}

The charged solution (4.1) was obtained by applying the charging procedure given in section 2 to the solution (3.4) that we obtained in section 3. This procedure added electric charge to any stationary solution of five-dimensional minimal supergravity, by means of an $O(1,1)$ duality transformation of its timelike dimensional reduction to four dimensions.

Another way of adding charge in five dimensions, which moreover allows the introduction of three independent charges in the $\mathcal{N}=2$ STU supergravity theory with $U(1)^{3}$ gauge fields, involves a thrice-repeated sequence of lifting to six dimensions, performing a Lorentz boost, and reducing again to $D=5$. In the intermediate stages, the discrete symmetries of the STU model are used twice over, to transfer the first and second charges from the Kaluza-

Klein vector of the $D=6$ to $D=5$ reduction to one of the other $U(1)$ fields. We have implemented this procedure in order to construct the generalisation of the solution (4.1) to the STU model.

We find that the generalised solution, with three independent electric charges, is given by

$$
\begin{aligned}
d s^{2}= & \left(H_{1} H_{2} H_{3}\right)^{1 / 3} d s_{4}^{2}-\frac{x y(d t+\omega)^{2}}{\left(H_{1} H_{2} H_{3}\right)^{2 / 3}} \\
d s_{4}^{2}= & \frac{x-y}{(1-x y)^{2}}\left[\frac{d x^{2}}{4 X}-\frac{d y^{2}}{4 Y}-\frac{X(d \phi+y d \psi)^{2}}{x(x-y)^{2}}+\frac{Y(d \phi+x d \psi)^{2}}{y(x-y)^{2}}\right] \\
\omega= & \frac{\mu c_{1} c_{2} c_{3}(d \phi+(x+y) d \psi)}{x y}-(\mu+q) s_{1} s_{2} s_{3}(d \phi(x+y)+x y d \psi) \\
& -\frac{q\left(c_{1}-s_{1} x\right)\left(c_{2}-s_{2} x\right)\left(c_{3}-s_{3} x\right)(d \phi+y d \psi)}{x(x-y)} \\
X= & (\mu+q)^{2}+a_{3} x+a_{2} x^{2}+a_{1} x^{3}+\mu^{2} x^{4} \\
Y= & \mu^{2}+a_{1} y+a_{2} y^{2}+a_{3} y^{3}+(\mu+q)^{2} y^{4}
\end{aligned}
$$

The gauge and scalar fields are given by

$$
\begin{aligned}
A_{i}= & \frac{c_{i} s_{i}(1-x y) d t+(\mu+q) c_{i} s_{j} s_{k}(d \phi(x+y)+x y d \psi)-\mu s_{i} c_{j} c_{k}(d \phi+(x+y) d \psi)}{H_{i}} \\
& -\frac{q\left(c_{i}-s_{i} y\right)\left(c_{j}-s_{j} x\right)\left(c_{k}-s_{k} x\right)(d \phi+y d \psi)}{(x-y) H_{i}}, \\
H_{i}= & c_{i}^{2}-s_{i}^{2} x y ; \quad i \neq j \neq k \text { and } i, j, k=1,2,3 .
\end{aligned}
$$

Here, we have defined $c_{i}=\cos \delta_{i}$ and $s_{i}=\sinh \delta_{i}$, where $\delta_{i}$ are the three boost parameters for the three electric charges. 
We have presented the solution in a symmetrical form for the $x$ and $y$ coordinates. The solution reduces to the solution (4.1) in minimal five-dimensional supergravity if the three charges are set equal (i.e. $\delta_{1}=\delta_{2}=\delta_{3}=\delta$ ), and also $x$ is sent to $1 / x$.

\section{B Black Ring Limit}

In this appendix, we consider a limiting form of the general charged solutions (4.1), which gives rise to black ring spacetimes 2 This is obtained by making the rescalings

$$
\begin{aligned}
& x \rightarrow \epsilon^{2} x, \quad y \rightarrow \epsilon^{2} y \quad \psi \rightarrow \epsilon \psi, \quad \phi \rightarrow \epsilon \phi, \\
& q \rightarrow \epsilon^{-3} q, \quad \mu \rightarrow \epsilon \mu, \quad a_{i} \rightarrow a_{i} \epsilon^{2 i-2}, \quad t \rightarrow t+3 c s^{2} q \epsilon^{-1} \phi,
\end{aligned}
$$

and then sending $\epsilon$ to zero. In the process, we also discard a divergent pure gauge term in the $U(1)$ potential. This leads to the solution

$$
\begin{aligned}
d s^{2} & =\frac{H}{(x-y)^{2}}\left(\frac{x d x^{2}}{4 G(x)}-\frac{x d y^{2}}{4 G(y)}-G(x) d \phi^{2}+\frac{x G(y) d \psi^{2}}{y}\right)-\frac{y}{x H^{2}}(d t+\omega)^{2}, \\
A & =\frac{\sqrt{3}}{x H}\left(c s(x-y) d t+\left(q s^{3} y^{2}-c^{2} s(\mu-2 q x y)\right) d \psi+\left(c^{3} q x^{2}-c s^{2}(\mu-2 q x y)\right) d \phi\right), \\
\omega & =\left(\frac{c^{3} \mu}{y}-3 c s^{2} q y\right) d \psi+\left(\frac{s^{3} \mu}{x}-3 c^{2} s q x\right) d \phi,
\end{aligned}
$$

where the function $H$ is the same as that given by (4.1). We again reparameterise so that $G(\xi)$ is given by

$$
G(\xi)=q^{2}\left(\xi-\xi_{1}\right)\left(\xi-\xi_{2}\right)\left(\xi-\xi_{3}\right)\left(\xi-\xi_{4}\right),
$$

where the the four roots satisfy $\xi_{1} \xi_{2} \xi_{3} \xi_{4}=\mu^{2} / q^{2}$. Furthermore we require that $\xi_{1}<\xi_{2}<$ $0<\xi_{3}<\xi_{4}$. Since the solution is obtained from a scaling limit, it follows that there is a residual scaling symmetry which enables us to set, without loss of generality, $\xi_{2}=-1$. We introduce

$$
\eta \equiv \sqrt{-\xi_{1}}>1
$$

The $x$ and $y$ coordinates will be taken to lie in the ranges

$$
-\eta^{2} \leq x \leq-1, \quad-1 \leq y \leq \infty
$$

There is a region corresponding to asymptotic infinity located at $x=-1=y$. Outer and inner horizons are located at $y=\xi_{3}$ and $y=\xi_{4}$ respectively. In order for $g_{\psi \psi}$ and $g_{\phi \phi}$ to

\footnotetext{
${ }^{2}$ These black ring solutions are contained within those found in [14. We give a discussion for their global properties here because our methods of analysis are rather different from those employed in [14, and also we give some explicit results for the thermodynamic properties that did not appear in [14].
} 
be non-negative on the degenerate surfaces $x=-\eta^{2}, x=-1$ and $y=-1$, it is necessary to shift the time coordinate $t$, according to

$$
t \rightarrow t+c\left(c^{2} \mu-3 q s^{2}\right) \psi-s\left(3 c^{2} q-\mu s^{2}\right) \phi
$$

and also to impose the additional constraint

$$
s\left(\mu s^{2}+3 c^{2} q \eta^{2}\right)=0 .
$$

This leads to a bifurcation of solutions:

$$
s=0, \quad \text { or } \quad q=-\frac{\mu s^{2}}{3 c^{2} \eta^{2}} .
$$

The first case corresponds to turning off the electric charge. The second case has a nonvanishing electric charge, related to the dipole charge. We shall discuss the two cases separately.

\section{B.1 Dipole ring}

This solution can be found in [13] in a different coordinate system. In this case, we turn off the electric charges by setting $s=0$, and hence $c=1$. The Killing vector $\partial / \partial \phi$ degenerates at both $x=\xi_{1} \equiv-\eta^{2}$ and $x=\xi_{2}=-1$. For the two resulting periodicity conditions to be consistent, it is necessary that the parameters in the solution be chosen such that

$$
q^{2}=\frac{\mu^{2}\left(\xi_{3}-\eta\right)}{\eta^{3} \xi_{3}\left(\xi_{3}+1+\eta+\eta^{2}\right)} .
$$

Thus we see that $\xi_{3} \geq \eta$. Furthermore, since we have $\xi_{4} \geq \xi_{3}$, it follows that the parameter $\xi_{3}$ lies in the range

$$
\eta \leq \xi_{3} \leq \eta+(1+\eta) \sqrt{\eta} .
$$

The lower bound corresponds to the neutral black ring solution, whilst the upper bound corresponds to an extremal black ring.

The Killing vector $\partial / \partial \psi$ degenerates at $y=-1$. This determines the periodicity of $\psi$. Ii is convenient to rescale both the azimuthal coordinates $\phi$ and $\psi$, according to

$$
\phi=\nu \tilde{\phi}, \quad \psi=\nu \tilde{\psi}
$$

where

$$
\nu=\frac{\eta^{3} \xi_{3}\left(1+\eta+\eta^{2}+\xi_{3}\right)}{\mu^{2}(\eta-1)(\eta+1)^{2}\left(\eta^{2}+\xi_{3}\right)\left(1+\xi_{3}\right)} .
$$

Then, $\tilde{\phi}$ and $\tilde{\psi}$ have independent $2 \pi$ periods (i.e. they are defined on a square lattice of side $2 \pi$ ). With this condition, our solution is regular without naked CTCs outside the horizon. 
The asymptotic region at infinity is located at $x=y=-1$. This may be seen by making the coordinate transformations

$$
x=-1-\frac{\nu \cos ^{2} \theta}{r^{2}}, \quad y=-1+\frac{\nu \sin ^{2} \theta}{r^{2}} .
$$

At large $r$, the metric then aproaches

$$
d s^{2} \sim-d t^{2}+d r^{2}+r^{2}\left(d \theta^{2}+\cos ^{2} \theta d \tilde{\phi}^{2}+\sin ^{2} \theta d \tilde{\psi}^{2}\right) .
$$

By evaluating Komar integrals at infinity, we find that the mass and the angular momenta are given by

$$
M=\frac{3}{8} \pi \nu, \quad J_{\tilde{\psi}}=\frac{1}{4} \pi \mu \nu^{2}, \quad J_{\tilde{\phi}}=0 .
$$

The horizon is located at $y=\xi_{3}$. The Killing vector

$$
\ell=\frac{\partial}{\partial t}+\Omega_{\tilde{\psi}} \frac{\partial}{\partial \tilde{\psi}}
$$

becomes null on the horizon, where $\Omega_{\tilde{\psi}}$, the angular velocity at the horizon, is given by

$$
\Omega_{\tilde{\psi}}=-\frac{\xi_{3}}{\mu \nu\left(1+\xi_{3}\right)} .
$$

By calculating the surface gravity $\kappa$ at the horizon, we find that the Hawking temperature $T=\kappa /(2 \pi)$ is given by

$$
T=\frac{\mu\left(\xi_{3}+\eta^{2}\right)\left(\eta(1+\eta)^{2}-\left(\xi_{3}-\eta\right)^{2}\right)}{2 \pi \eta^{3} \sqrt{\xi_{3}}\left(1+\eta+\eta^{2}+\xi_{3}\right)},
$$

It is straightforward to see that the horion has the topology $S^{2} \times S^{1}$, and that the entropy, equal to one quarter of the horizon area, is

$$
S=\frac{\pi^{2} \mu \nu^{2}\left(\eta^{2}-1\right)}{2 \sqrt{\xi_{3}}\left(\eta^{2}+\xi_{3}\right)} .
$$

The solution is supported purely by a magnetic dipole charge, given by

$$
\mathcal{D}=\frac{1}{8} \int F=\frac{1}{4} \pi \sqrt{3} q \nu\left(\eta^{2}-1\right),
$$

where the integration is performed over the $S^{2}$ component of the horizon. The potential difference between the horizon and infinity is calculated by Hodge dualising the magnetic 2-form $F$ to an electric 3-form $G=* F=d B+A \wedge F / \sqrt{3}$. The dipole potential $\Phi_{\mathcal{D}}$ is given by the difference between $B_{\tilde{\psi} t}$ at the horizon and infinity, and so

$$
\Phi_{\mathcal{D}}=\sqrt{3} q \nu\left(\xi_{3}+1\right)
$$


With these quantities, we find that the first law of thermodynamics is satisfied. In particular, we have

$$
d M=T d S+\Omega_{\psi} d J_{\psi}+\Phi_{\mathcal{D}} d \mathcal{D}, \quad M=\frac{3}{2} T S+\frac{3}{2} \Omega_{\psi} J_{\psi}+\frac{1}{2} \Phi_{\mathcal{D}} \mathcal{D}
$$

(See [19] for a detailed discussion of the role of magnetic dipole charge in black hole thermodynamics.)

There exists an extremal limit when $\xi_{3}=\xi_{4}$, which implies that

$$
\xi_{3}=\eta+(1+\eta) \sqrt{\eta}
$$

In this limit, the temperature goes to zero, and the near horizon geometry becomes a warped product of a distorted $\mathrm{AdS}_{3}$ and $S^{2}$. There is a decoupling limit which reduces the solution to its near horizon geometry, analogous to the $\mathrm{AdS}_{5} \times S^{5}$ decoupling limit of the D3-brane. Letting $y=\xi_{3}-\epsilon r$ and $t \rightarrow t / \epsilon$, and sending $\epsilon$ to zero, we have

$$
\begin{aligned}
d s^{2}= & \frac{\eta^{2} \xi_{3} x d x^{2}}{4 \mu^{2}(x+1)\left(x+\eta^{2}\right)\left(x-\xi_{3}\right)^{4}}-\frac{\eta^{2} \xi_{3}^{2}(x+1)\left(x+\eta^{2}\right) d \phi^{2}}{\mu^{2}(\sqrt{\eta}-1)^{2}\left(1+\xi_{3}\right)^{6}} \\
& -\frac{\eta^{3} \xi_{3}^{2}}{4 \mu^{2}\left(1+\xi_{3}\right)^{4} x}\left(d \psi^{\prime}+r d t^{\prime}\right)^{2}-\frac{\eta^{2} \xi_{3}^{2} x}{4 \mu^{2}\left(1+\xi_{3}\right)\left(\eta^{2}+\xi_{3}\right)\left(x-\xi_{3}\right)^{2}}\left(\frac{d r^{2}}{r^{2}}+r^{2} d t^{\prime 2}\right) \\
A= & \frac{\sqrt{3} \eta \xi_{3} x}{\mu(\sqrt{\eta}-1)\left(1+\xi_{3}\right)^{3}} d \phi
\end{aligned}
$$

where

$$
t^{\prime}=\frac{2 \mu\left(\eta^{2}+\xi_{3}\right)}{\eta^{2} \xi_{3}^{3 / 2}} t . \quad \psi^{\prime}=\frac{\sqrt{\eta}-1}{2 \sqrt{\eta \xi_{3}}}\left(\psi-\Omega_{\psi} t\right) .
$$

The first two terms in the metric in (B.24) give rise to an $S^{2}$, whilst the remaining terms describe a homogeneously squashed $\mathrm{AdS}_{3}$, viewed as a $U(1)$ bundle over $\mathrm{AdS}_{2}$.

\section{B.2 Electrically-charged dipole ring}

This solution can be found in [14] in a different coordinate system. The solution corresponds to the second choice in (B.8), which requires that the parameters determining the electric charge and the dipole charge are related by

$$
q=-\frac{\mu s^{2}}{3 c^{2} \eta^{2}}
$$

The compatibility between the periodicity conditions on $\phi$ at the two degeneration surfaces $x=-\eta^{2}$ and $x=-1$ implies that the parameters must be chosen such that

$$
\frac{\left(\eta^{2}+\xi_{3}\right)\left(\eta^{2}+\xi_{4}\right)}{\eta\left(1+\xi_{3}\right)\left(1+\xi_{4}\right)}=1
$$


It is convenient again to rescale the azimuthal coordinates $\phi$ and $\psi$, according to $\phi=\nu \tilde{\phi}$ and $\psi=\nu \tilde{\psi}$, where now

$$
\nu=\frac{1}{q^{2}\left(\eta^{2}-1\right)\left(1+\xi_{3}\right)\left(1+\xi_{4}\right)} .
$$

We then find that $\tilde{\phi}$ and $\tilde{\psi}$ have independent $2 \pi$ periods, and are thus defined on a square lattice of side $2 \pi$.

Asymptotic infinity is at $x=y=-1$. To see this explicitly, we can make the same

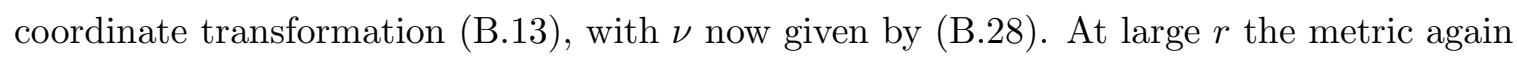
approaches (B.14). From the aymptotic form of the solution we find that the mass $M$, electric charge $Q_{e}$ and angular momenta $J_{\tilde{\phi}}$ and $J_{\tilde{\psi}}$ are given by

$$
\begin{aligned}
& M=\frac{3}{8} \pi\left(1+2 s^{2}\right) \nu, \quad Q_{e}=\frac{1}{4} \sqrt{3} \pi s c \nu \\
& J_{\tilde{\psi}}=\frac{1}{4} \pi c\left(\mu c^{2}+3 q s^{2}\right) \nu^{2}, \quad J_{\tilde{\phi}}=\frac{1}{4} \pi s\left(\mu s^{2}+3 q c^{2}\right) \nu^{2} .
\end{aligned}
$$

The horizon is located at $y=\xi_{3}$, and the corresponding Killing vector that vanishes on the horizon is given by

$$
\ell=\frac{\partial}{\partial t}-\Omega_{\tilde{\psi}} \frac{\partial}{\partial \tilde{\psi}}
$$

where $\Omega_{\tilde{\psi}}$ is the angular velocity, given by

$$
\Omega_{\tilde{\psi}}=\frac{\xi_{3}}{\nu c\left(1+\xi_{3}\right)\left(\mu c^{2}-3 q s^{2} \xi_{3}\right)} .
$$

(Note that $\Omega_{\tilde{\phi}}=0$, even though $J_{\tilde{\phi}}$ is non-zero.) The temperature is given by

$$
T=\frac{q^{2} \sqrt{\xi_{3}}\left(\eta^{2}+\xi_{3}\right)\left(\xi_{4}-\xi_{3}\right)}{2 \pi c\left(c^{2} \mu-3 q s^{2} \xi_{3}\right)} .
$$

The horizon can be seen to have the topology $S^{2} \times S^{1}$, and by calculating its area, we find that the entropy is

$$
S=\frac{c \nu^{2} \pi^{2}\left(\eta^{2}-1\right)\left(c^{2} \mu-3 q s^{2} \xi_{3}\right)}{2 \sqrt{\xi_{3}}\left(\eta^{2}+\xi_{3}\right)} .
$$

The electrostatic potential difference between horizon and infinity is given by

$$
\Phi_{e}=\frac{\sqrt{3} s\left(q \xi_{3}+c^{2}\left(\mu-3 q \xi_{3}\right)\right)}{c\left(3 q \xi_{3}+c^{2}\left(\mu-3 q \xi_{3}\right)\right)} .
$$

The magnetic dipole charge is given by

$$
\mathcal{D}=\frac{1}{8} \int F=\frac{3 \sqrt{3} \pi s^{2} c^{3} \eta^{2} \xi_{3}}{2 \mu\left(1+\xi_{3}\right)\left(9 c^{4} \eta^{2}+s^{4} \xi_{3}\right)} .
$$

This is related to $J_{\tilde{\phi}}$ and the electric charge $Q_{e}$ by

$$
J_{\tilde{\phi}}=\frac{2}{\pi} Q_{e} \mathcal{D}
$$


From the expected generalised Smarr relation

$$
M=\frac{3}{2} T S+\frac{3}{2} \Omega_{\tilde{\psi}} J_{\tilde{\psi}}+\Phi_{e} Q_{e}+\frac{1}{2} \Phi_{\mathcal{D}} \mathcal{D}
$$

we find that the dipole potential is given by

$$
\Phi_{\mathcal{D}}=\frac{\sqrt{3} s^{2} c \eta^{2} \xi_{3}}{2 \mu\left(\eta^{2}-1\right)}\left[\frac{3}{9 c^{4} \eta^{2}+s^{4} \xi_{3}}-\frac{1}{c^{4} \eta^{2}+s^{4} \xi_{3}}\right] .
$$

It can then be verified that the first law

$$
d M=T d S+\Omega_{\tilde{\psi}} d J_{\tilde{\psi}}+\Phi_{e} d Q_{e}+\Phi_{\mathcal{D}} d \mathcal{D}
$$

is satisfied.

\section{References}

[1] R. Emparan and H.S. Reall, A rotating black ring in five dimensions, Phys. Rev. Lett. 88, 101101 (2002), hep-th/0110260.

[2] A.A. Pomeransky and R.A. Sen'kov, Black ring with two angular momenta, hep-th/0612005.

[3] H. Lü, Jianwei Mei and C.N. Pope, New black holes in five dimensions, arXiv:0804.1152 [hep-th].

[4] J.F. Plebanski and M. Demianski, Rotating, charged, and uniformly accelerating mass in general relativity, Annals Phys. 98 (1976) 98.

[5] W. Chen, H. Lü and C.N. Pope, Kerr-de Sitter black holes with NUT charges, Nucl. Phys. B762, 38 (2007), hep-th/0601002.

[6] W. Chen, H. Lü and C.N. Pope, General Kerr-NUT-AdS metrics in all dimensions, Class. Quant. Grav. 23, 5323 (2006), hep-th/0604125.

[7] G.W. Gibbons, H. Lü, D.N. Page and C.N. Pope, The general Kerr-de Sitter metrics in all dimensions, J. Geom. Phys. 53, 49 (2005), hep-th/0404008.

[8] G.W. Gibbons, H. Lü, D.N. Page and C.N. Pope, Rotating black holes in higher dimensions with a cosmological constant, Phys. Rev. Lett. 93, 171102 (2004), hep-th/0409155.

[9] R.C. Myers and M.J. Perry, Black holes in higher dimensional space-times, Annals Phys. 172: 304 (1986). 
[10] D. Kubiznak and P. Krtous, On conformal Killing-Yano tensors for PlebanskiDemianski family of solutions, Phys. Rev. D76, 084036 (2007), arXiv:0707.0409 [gr-qc].

[11] M. Cvetič and D. Youm, General rotating five dimensional black holes of toroidally compactified heterotic string, Nucl. Phys. B476, 118 (1996), hep-th/9603100.

[12] Z.W. Chong, M. Cvetič, H. Lü and C.N. Pope, General non-extremal rotating black holes in minimal five-dimensional gauged supergravity, Phys. Rev. Lett. 95, 161301 (2005) hep-th/0506029.

[13] R. Emparan, Rotating circular strings, and infinite non-uniqueness of black rings, JHEP 0403, 064 (2004), hep-th/0402149.

[14] H. Elvang, R. Emparan and P. Figueras, Non-supersymmetric black rings as thermally excited supertubes, JHEP 0502, 031 (2005), hep-th/0412130.

[15] S. Mizoguchi and N. Ohta, More on the similarity between $D=5$ simple supergravity and $M$ theory, Phys. Lett. B441, 123 (1998), hep-th/9807111.

[16] E. Cremmer, B. Julia, H. Lü and C.N. Pope, Higher-dimensional origin of D $=3$ coset symmetries, hep-th/9909099.

[17] A. Bouchareb, G. Clement, C.M. Chen, D.V. Gal'tsov, N.G. Scherbluk and T. Wolf, $G_{2}$ generating technique for minimal $D=5$ supergravity and black rings, Phys. Rev. D76, 104032 (2007), arXiv:0708.2361 [hep-th].

[18] M. Cvetič, H. Lü, D.N. Page and C.N. Pope, New Einstein-Sasaki spaces in five and higher dimensions, Phys. Rev. Lett. 95, 071101 (2005), hep-th/0504225.

[19] K. Copsey and G.T. Horowitz, The role of dipole charges in black hole thermodynamics, Phys. Rev. D73, 024015 (2006), hep-th/0505278. 